\title{
ON A CLASS OF DIFFERENTIAL-DIFFERENCE OPERATORS IN SPACES OF ANALYTIC FUNCTIONS
}

\author{
STEPAN LINCHUK AND YURIY LINCHUK
}

\begin{abstract}
We define the differential-difference operator which generalizes the Dunkl operator and the Bessel-Struve operator in the space of analytic functions in an arbitrary starlike $p$ symmetric domain of the complex plane with respect to the origin, where $p$ is some positive integer. We investigate conditions of the equivalence of this operator to the power of the usual differentiation. We describe the commutant of this operator. We establish the hypercyclicity and the chaoticity of this operator. We investigate properties of diagonal operators induced by the Taylor coefficients of the generalized hypergeometric function.
\end{abstract}

Mathematics subject classification (2010): 47B38.

Keywords and phrases: Dunkl operator, Bessel-Struve operator, space of analytic functions, transmutation operator, diagonal operator, hypercyclic operator.

\section{REFERENCES}

[1] F. Bayart, E. Matheron, Dynamics of linear operator, Cambridge Tracts in Mathematics, 179, Cambridge University Press, Cambridge, 2009.

[2] N. Ben SALEM, S. KALlEL, Integro-differential-difference equations associated with the Dunkl operator and entire functions, Comment. Math. Univ. Carolinae. 45, 4 (2004), 699-725.

[3] L. Bernal-Gonzalez, A. Bonilla, Rate of growth of hypercyclic and frequently hypercyclic functions for the Dunkl Operator, Mediterr. J. Math. 13, 5 (2016), 3359-3372.

[4] J. J. BETANCOR, M. SiFI, K. TRIMECHE, Hypercyclic and chaotic convolution operators associated with the Dunkl operator on $\mathbb{C}$, Acta Math. Hung. 106, (1-2) (2005), 101-116.

[5] A. V. BRATiShCHev, Chaotic commutating with the Dunkl differentiation linear operators on the space of analytic functions, Vestnik of DSTU. 9, 2 (2009), 196-207, (in Russian).

[6] J. DELSARTE, Sur certaines transformations fonctionelles relative aux equations linearines aux derives partielles du second ordre, C. R. Math. Acad. Sci. Paris. 206, (1938), 178-182.

[7] J. Dels arte, J. L. Lions, Transmutations d'operateurs differentieles dans le domaine complexe, Comm. Math. Helv. 32, 2 (1957), 113-128.

[8] I. H. Dimovs Ki, Convolutional Calculus, Kluwer, Dordrecht, 1990.

[9] C. F. DunKL, Differential difference operators associated to reflection groups, Trans. Amer. Math. Soc. 311, (1989), 167-183.

[10] A. GASMI, M. SIFI, The Bessel-Struve intertwining operator on $\mathbb{C}$ and mean-periodic functions, Int. J. Math. Sci. 59, (2004), 3171-3185.

[11] A. GASMI, M. SIFI, Analytic mean-periodic functions associated with the Bessel-Struve operator on a disk, Global J. Pure and Appl. Math. 1, 1 (2005), 55-68.

[12] S. Hamem, L. Kamoun, S. Negzaoui, Cowling-Price type theorem related to Bessel-Struve transform, Arab J. Math. Sci. 19, 2 (2013), 187-198.

[13] L. Kamoun, M. Sifi, Bessel-Struve intertwining operator and generalized Taylor series on the real line, Integral Transforms and Spec. Funct. 16, 1 (2005), 39-55.

[14] YU. S. LINCHUK, Generalized Dunkl-Opdam operator and its properties in the spaces of functions analytic in domains, J. Math. Sci. 220, 1 (2017), 1-14.

[15] S. NegZaoui, Beurling-Hormander's theorem related to Bessel-Struve transform, Integral Transforms Spec. Funct. 27, 9 (2016), 685-697. 
[16] N. B. SAlEm, S. KALlel, Mean-periodic functions associated with the Dunkl operators, Integral Transforms Spec. Funct. 15, 2 (2004), 155-179.

[17] M. YU. TSAR' KOV, Isomorphismen einiger analytischer Raume, welche mit dem Grad des Differentiations operators kommutieren, Teor. Funkts., Funkts. Anal. Prilozh. 11, (1970), 86-92. 